\title{
FASTKD2 and human memory: functional pathways and prospects for novel therapeutic target development for Alzheimer's disease and age-associated memory decline
}

\author{
Vijay K Ramanan and \\ Center for Neuroimaging, Department of Radiology \& Imaging Sciences, Indiana University \\ School of Medicine, Indianapolis, IN, USA; Department of Medical \& Molecular Genetics, Indiana \\ University School of Medicine, IN, USA; Medical Scientist Training Program, Indiana University \\ School of Medicine, Indianapolis, IN, USA; Indiana Alzheimer Disease Center, Indiana University \\ School of Medicine, Indianapolis, IN, USA
}

\section{Andrew J Saykin}

Center for Neuroimaging, Department of Radiology \& Imaging Sciences, Indiana University School of Medicine, 355 West 16th Street, Suite 4100, Indianapolis, IN 46202, USA; Department of Medical \& Molecular Genetics, Indiana University School of Medicine, IN, USA; Medical Scientist Training Program, Indiana University School of Medicine, Indianapolis, IN, USA; Indiana Alzheimer Disease Center, Indiana University School of Medicine, Indianapolis, IN, USA Tel.: +1 3179637501 Fax: +13179637547

Andrew J Saykin: asaykin@iupui.edu

\section{Keywords}

Alzheimer's disease; apoptosis; cognitive aging; drug target; FASTKD2; fas-associated serine/ threonine kinase domains 2; functional genomics; inflammation; memory; microRNA; mitochondria

\begin{abstract}
Impairment in episodic memory is typically the earliest clinical deficit to appear in Alzheimer's disease (AD), the most common cause of dementia and a source of immense personal and societal burden. Unfortunately, the mechanisms underlying AD and other agerelated conditions causing cognitive deficits are only partially understood, limiting the development of disease-modifying therapies and novel early diagnostic biomarkers.

Recently, we reported the discovery of a SNP in the FASTKD2 gene associated with better memory performance in a large sample of older Americans [1]. We also demonstrated that this new memory-protective SNP was associated with increased volume and gray matter density in the hippocampus, a key brain structure for encoding and retrieving memories that
\end{abstract}

\footnotetext{
(C) 2015 Future Medicine Ltd

Correspondence to: Andrew J Saykin, asaykin@iupui . edu.

Financial \& competing interests disclosure: The authors have no other relevant affiliations or financial involvement with any organization or entity with a financial interest in or financial conflict with the subject matter or materials discussed in the manuscript apart from those disclosed.

No writing assistance was utilized in the production of this manuscript
} 
is among the earliest regions affected by AD. Shortly thereafter, a separate report from an independent group identified perturbations in FASTKD2 expression in brain astrocytes derived from postmortem tissue samples from AD patients [2].

Prior to these studies, FASTKD2 had not been linked to cognition or AD. This new evidence obtained through diverse methodologies and analytical strategies suggests that FASTKD2 may have potential as a novel target for biomarker and drug development against AD and age-associated cognitive decline. As a result, this is an opportune moment to critically appraise extant knowledge about FASTKD2 and its functional pathways in order to guide next steps aimed at translating mechanistic knowledge into potential clinical strategies.

\section{FASTKD protein family}

FASTKD2 encodes one of a family of proteins (including FASTK and FASTKD1-5) that share a common structure including a mitochondrial targeting domain, multiple serine/ threonine kinase domains and an RNA-binding domain [3]. Much of the early literature on this protein family focuses on FASTK, which constitutively inhibits apoptosis when tethered to the outer mitochondrial membrane but promotes apoptosis when released to the cell cytoplasm following activation of the Fas/CD95 'death receptor' [3,4]. The proapoptotic function of FASTK is mediated by its binding and modulating the function of TIA1, an mRNA-binding protein that normally silences the production of apoptotic and inflammatory mediators including TNF-a [5].

As discussed below, initial studies of the other FASTKD protein family members indicate broadly shared functions with FASTK. This evidence is not surprising given the common structure and mitochondrial localization of these proteins. However, a closer examination of these putative functional pathways can provide mechanistic clues to characterize the particular impact of FASTKD2 on brain structure and function (Supplementary Figure 1; see online at: www.future-medicine.com/doi/suppl/10.2217/pgs.15.8).

\section{FASTKD2 \& apoptosis}

The first functional study of FASTKD2 ensued following the discovery of gene mutations causing a rare mitochondrial encephalomyopathy [6]. Due to its FASTK domain, the authors hypothesized a role for the FASTKD2 protein in apoptosis and found that on treatment with staurosporine (a well-described inducer of apoptosis), cells with loss-of-function FASTKD2 mutations exhibited less apoptosis while cells with FASTKD2 overexpression exhibited more apoptosis [6].

Subsequent studies identified FASTKD2 as a vital part - along with NRIF3 and DIF1 - of a 'death switch' modulating apoptosis in cancer cells [7,8]. Further insight can also be found in the broader interaction network for the FASTKD2 protein, which includes numerous proapoptotic factors including TRAF6, TSC22D1 and c-Myc [9].

In our report, we demonstrated that the novel FASTKD2 SNP associated with better memory performance was also associated with lower levels in cerebrospinal fluid of four proteins involved in fas-mediated apoptosis [1]. Although it was beyond the scope of our manuscript 
to directly assess the impact of this SNP on activation of apoptosis at the cellular level, this additional finding suggests that modulation of cell death pathways may account for the neuroprotective effect of FASTKD2 on memory.

\section{FASTKD2 \& mitochondrial function}

The protein encoded by FASTKD2 localizes to the inner mitochondrial membrane and is expressed at its highest levels in the richly energy-dependent tissues of the brain [6,10]. A nonsense mutation in FASTKD2 is known to underlie respiratory chain complex IV (cytochrome c oxidase) deficiency, a rare and typically fatal disorder that presents in infancy with developmental delay, myopathy and encephalopathy including demyelinating brain lesions and epilepsy [6,11]. Recently, missense FASTKD2 mutations were also identified in patients with inherited ataxias, a heterogeneous group of disorders with frequent mitochondrial origin [12].

It is not yet known how these FASTKD2 mutations lead to their cellular and clinical phenotypes. For example, while FASTKD2 may be directly involved in the synthesis, localization and/or function of cytochrome c oxidase, it is also possible that knocking out FASTKD2 starts a chain of events yielding broader mitochondrial dysfunction negatively impacting the respiratory chain and its central role in cellular energetics. Indeed, the protein encoded by family member FASTKD3 is known to interact with both respiratory chain components and mitochondrial DNA translation machinery [3], suggesting broad potential functionality of this protein class in the cellular powerhouse. Further study will be needed to better understand the pathogenic sequence initiated by rare mutations as well as more common genetic variants.

Beyond these findings in rare genetic conditions, mitochondrial dysfunction and closely related oxidative stress pathways are major candidate mechanisms proposed to underlie neurodegeneration in aging and disease [13,14]. The new identification of expression changes in FASTKD2 and other mitochondrial genes in AD brain cells burnishes this hypothesis and enhances the foundation for additional functional genomics studies to clarify underlying mechanisms [2].

\section{FASTKD2 \& inflammation}

Despite the mitochondrial localization of its encoded protein, there is an increasing body of work suggesting a proinflammatory role for FASTKD2. A likely mechanism for this effect may be related to the FASTK domain and its inhibition of TIA1, ultimately promoting production of the systemic inflammation mediator TNF-a [15,16]. The Fas/FasL axis is itself considered a strong regulator of inflammation and in some biological contexts may lead to tissue damage through inflammatory responses rather than through apoptosis [17]. This evidence may be particularly compelling in the setting of $\mathrm{AD}$, where inflammation and immune system activation are increasingly viewed as playing central roles in mediating and/or compensating for cellular stress induced by amyloid- $\beta$ deposition $[18,19]$. 


\section{Future directions}

Population genomics analyses of additional data sets, including other relevant AD endophenotypes, may provide further insight regarding the clinical impact of FASTKD2. Expression studies using microarray or RNA-seq data will also be useful to examine the relationship between specific genetic variants and canonical transcripts as well as splice variant and other alternative transcripts. Finally, deep sequencing of the FASTKD2 locus in large samples will facilitate characterization of regional linkage disequilibrium structure (including functional 'tag' SNPs), other types of variants (including rare, copy number and structural variants) and epigenetic modification sites.

Functional genomic studies will also be crucial to elucidate the basic mechanisms connecting these genetic variants to expression and biological action. The overlap of two microRNA genes (MIR 3130-1 and 2) with the intron containing the memory-associated SNP raises the possibility that variants in this region may regulate microRNA-mediated silencing of gene expression [1]. However, other leading possibilities for further exploration include potential dysfunction in the FASTKD2 RNA-binding domain [20] or at transcription factor or splicing machinery-binding sites. Although FASTKD2 appears to lack homologs in yeast, worms and flies [6], the creation of mammalian models and/or cell lines will be vital for this effort and could be crossed with analogous AD model systems to directly assess the impact of knockout, knockdown and overexpression of FASTKD2.

\section{Conclusion}

Along with related new discoveries and prior functional studies of this gene, the novel association of FASTKD2 with memory performance and hippocampal structure potentially opens new avenues for exploration of strategies to modulate neurodegeneration in AD and cognitive aging. Given that genes and proteins do not act in isolation [21], a better understanding of the range of biological activities for FASTKD2 will help focus efforts to develop potential disease-modifying drugs targeting these functional pathways.

\section{Supplementary Material}

Refer to Web version on PubMed Central for supplementary material.

\section{Acknowledgments}

The authors acknowledge the support of NIH R01AG19771, P30AG10133, R01LM011360 and R00LM011384; NSF IIS-1117335; and the following funding sources for the analyses discussed in this manuscript: The Health and Retirement Study (HRS) is sponsored by NIH grants U01AG009740, RC2AG036495 and RC4AG039029 and is conducted by the University of Michigan. Further information can be found at http://hrsonline.isr.umich.edu/ index.php. The Alzheimer's Disease Neuroimaging Initiative (ADNI) is funded by NIH grants U01AG024904, P30AG010129, K01AG030514 and U24AG21886; Department of Defense award number W81XWH-12-2-0012; the National Institute of Biomedical Imaging and Bioengineering and through generous private sector contributions facilitated by the Foundation for the NIH. The Canadian Institutes of Health Research supports ADNI clinical sites in Canada. The Indiana Memory and Aging Study (IMAS) is supported in part by NIH grants R01AG19771, P30AG10133, U54 RR025761, RR027710-01 and RR020128. 


\section{References}

1. Ramanan VK, Nho K, Shen L, et al. FASTKD2 is associated with memory and hippocampal structure in older adults. Mol Psychiatry. 2014 Epub ahead of print. 10.1038/mp.2014.142

2. Sekar S, Mcdonald J, Cuyugan L, et al. Alzheimer's disease is associated with altered expression of genes involved in immune response and mitochondrial processes in astrocytes. Neurobiol Aging. 2014; 36(2):583-591. [PubMed: 25448601]

3. Simarro M, Gimenez-Cassina A, Kedersha N, et al. Fast kinase domain-containing protein 3 is a mitochondrial protein essential for cellular respiration. Biochem Biophys Res Commun. 2010; 401(3):440-446. [PubMed: 20869947]

4. Li W, Simarro M, Kedersha N, Anderson P. FAST is a survival protein that senses mitochondrial stress and modulates TIA-1-regulated changes in protein expression. Mol Cell Biol. 2004; 24(24): 10718-10732. [PubMed: 15572676]

5. Phillips K, Kedersha N, Shen L, Blackshear PJ, Anderson P. Arthritis suppressor genes TIA-1 and TTP dampen the expression of tumor necrosis factor alpha, cyclooxygenase 2, and inflammatory arthritis. Proc Natl Acad Sci USA. 2004; 101(7):2011-2016. [PubMed: 14769925]

6. Ghezzi D, Saada A, D'Adamo P, et al. FASTKD2 nonsense mutation in an infantile mitochondrial encephalomyopathy associated with cytochrome c oxidase deficiency. Am J Hum Genet. 2008; 83(3):415-423. [PubMed: 18771761]

7. Das S, Yeung K, Mahajan M, Samuels H. Fas activated serine-threonine kinase domains 2 (FASTKD2) mediates apoptosis of breast and prostate cancer cells through its novel FAST2 domain. BMC Cancer. 2014; 14(1):852. [PubMed: 25409762]

8. Yeung KT, Das S, Zhang J, et al. A novel transcription complex that selectively modulates apoptosis of breast cancer cells through regulation of FASTKD2. Mol Cell Biol. 2011; 31(11): 2287-2298. [PubMed: 21444724]

9. Ewing RM, Chu P, Elisma F, et al. Large-scale mapping of human protein-protein interactions by mass spectrometry. Mol Syst Biol. 2007; 3:89. [PubMed: 17353931]

10. Kang HJ, Kawasawa YI, Cheng F, et al. Spatio-temporal transcriptome of the human brain. Nature. 2011; 478(7370):483-489. [PubMed: 22031440]

11. Ruhoy IS, Saneto RP. The genetics of Leigh syndrome and its implications for clinical practice and risk management. Appl Clin Genet. 2014; 7:221-234. [PubMed: 25419155]

12. Pyle A, Smertenko T, Bargiela D, et al. Exome sequencing in undiagnosed inherited and sporadic ataxias. Brain. 2014; 138(Pt 2):276-283. [PubMed: 25497598]

13. Ramanan VK, Saykin AJ. Pathways to neurodegeneration: mechanistic insights from GWAS in Alzheimer's disease, Parkinson's disease, and related disorders. Am J Neurodegener Dis. 2013; 2(3):145-175. [PubMed: 24093081]

14. Lin MT, Beal MF. Mitochondrial dysfunction and oxidative stress in neurodegenerative diseases. Nature. 2006; 443(7113):787-795. [PubMed: 17051205]

15. Simarro M, Giannattasio G, De La Fuente MA, et al. Fas-activated serine/threonine phosphoprotein promotes immune-mediated pulmonary inflammation. J Immunol. 2010; 184(9): 5325-5332. [PubMed: 20363972]

16. Piecyk M, Wax S, Beck AR, et al. TIA-1 is a translational silencer that selectively regulates the expression of TNF-alpha. EMBO J. 2000; 19(15):4154-4163. [PubMed: 10921895]

17. Bird L. Inflammation Travelling Fast. Nat Rev Immunol. 2010; 10(4):219.

18. Prinz M, Priller J, Sisodia SS, Ransohoff RM. Heterogeneity of CNS myeloid cells and their roles in neurodegeneration. Nat Neurosci. 2011; 14(10):1227-1235. [PubMed: 21952260]

19. Lucin KM, Wyss-Coray T. Immune activation in brain aging and neurodegeneration: too much or too little? Neuron. 2009; 64(1):110-122. [PubMed: 19840553]

20. Castello A, Fischer B, Eichelbaum K, et al. Insights into RNA biology from an atlas of mammalian mRNA-binding proteins. Cell. 2012; 149(6):1393-1406. [PubMed: 22658674]

21. Ramanan VK, Shen L, Moore JH, Saykin AJ. Pathway analysis of genomic data: concepts, methods, and prospects for future development. Trends Genet. 2012; 28(7):323-332. [PubMed: 22480918] 